\title{
Unsymmetrical Photodimerization of a 9-Aminomethylanthracene in the Crystalline Salt
}

\author{
Masahiro Horiguchi and Yoshikatsu Ito* \\ Department of Synthetic Chemistry and Biological Chemistry, Graduate School of Engineering, \\ Kyoto University, Katsura, Kyoto 615-8510, Japan \\ yito@sbchem.kyoto-u.ac.jp
}

\section{CONTENTS}

1. Instruments

page S1

2. Preparation of 9-(N,N-Dimethylaminomethyl)anthracene (1)

page $\mathrm{S} 1$

3. Preparation of Crystalline Salts

page S2

4. Spectral Data of the Photochemical Products

page 55

5. Dissociation Kinetics of usy-ht-2

page $\mathrm{S7}$

6. X-ray Crystal Structure Analysis

page $\mathrm{S7}$

7. ${ }^{1} \mathrm{H}$ and ${ }^{13} \mathrm{C}$ NMR Charts of usy-ht-2

page $\mathrm{S} 9$

\section{Instruments}

${ }^{1} \mathrm{H}$ NMR spectra were recorded on a $400 \mathrm{MHz}$ or $300 \mathrm{MHz}$ FT-NMR spectrometer. ${ }^{13} \mathrm{C}$ NMR spectra were recorded at $75 \mathrm{MHz}$. IR spectra were recorded on a FT-IR spectrometer. Mass spectra were recorded on a FAB or GC-EI spectrometer. Melting points were not corrected. Preparative thin-layer chromatography was carried out on laboratory-made silica gel plates.

\section{Preparation of 9-(N,N-Dimethylaminomethyl)anthracene (1) ${ }^{1,2}$}

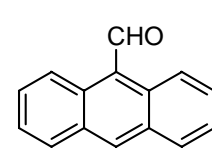

S1 (i) $\mathrm{Me}_{2} \mathrm{NH}_{2} \mathrm{Cl}, \mathrm{Ti}(\mathrm{O}-\mathrm{PPr})_{4}, \mathrm{TEA}, \mathrm{EtOH}, \mathrm{rt}$ 10h (ii) $\mathrm{NaBH}_{4}$, rt $14 \mathrm{~h}$

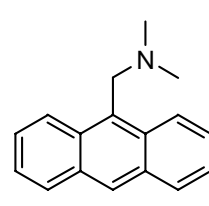

1

${ }^{1}$ Bhattacharyya, S. J. Org. Chem. 1995, 60, 4928-4929.

2 Ihmels, H.; Leusser, D.; Pfeiffer, M.; Stalke, D. Tetrahedron 2000, 56, 6867-6875. 
Dimethylammonium chloride (8.1 g, $99 \mathrm{mmol}$ in $50 \mathrm{~mL}$ of dehyd. EtOH), titanium tetraisopropoxide (29.2 mL, $99 \mathrm{mmol})$, and 9-anthraldehyde $(10.2 \mathrm{~g}, 50 \mathrm{mmol}$ in $700 \mathrm{~mL}$ of dehyd. EtOH) were successively added to a solution of triethylamine (10.0 g, $99 \mathrm{mmol})$ in dehyd. EtOH (10 $\mathrm{mL}$ ). After stirring for $10 \mathrm{~h}$ at rt, $\mathrm{NaBH}_{4}$ (2.85 g, $75 \mathrm{mmol}$ ) was added and the mixture was stirred for additional $14 \mathrm{~h}$ at rt. The reaction mixture was treated with an aqueous $\mathrm{NH}_{3}$ solution $(2 \mathrm{~N}, 160 \mathrm{~mL}$ ) and the precipitated solid was filtered and was washed with $\mathrm{CHCl}_{3}$. The filtrate and the washings were combined and evaporated. Then, after the residue was redissolved in $\mathrm{CHCl}_{3}$, conc. $\mathrm{HCl}$ (10 $\mathrm{mL}$ ) was added. The resultant precipitate was filtered and recrystallized from $\mathrm{MeOH} / \mathrm{AcOEt}$ to give colorless needles of the hydrochloride salt of 1 (12.7 g, $47 \mathrm{mmol}, 95 \%)$.

1•HCl: mp 248-250 ${ }^{\circ} \mathrm{C}$ (dec.) (lit. $\left.{ }^{3} \mathrm{mp} 252-254{ }^{\circ} \mathrm{C}\right) .{ }^{1} \mathrm{H}$ NMR $\left(\mathrm{CD}_{3} \mathrm{OD}\right) \delta 2.98$ (s, $\left.6 \mathrm{H}\right), 5.46$ (s, 2 H), 7.59 (ddd, $J=8.4,6.3,0.6 \mathrm{~Hz}, 2 \mathrm{H}$ ), 7.74 (ddd, $J=9.3,6.6,1.2 \mathrm{~Hz}, 2 \mathrm{H}$ ), 8.11 (d, $J=8.7 \mathrm{~Hz}, 2 \mathrm{H}$ ), 8.42 (dd, $J=9.0,0.9 \mathrm{~Hz}, 2 \mathrm{H}), 8.74$ (s, $1 \mathrm{H}$ ). ${ }^{13} \mathrm{C}$ NMR $\left(\mathrm{CD}_{3} \mathrm{OD}\right) \delta 43.8,53.9,121.5,124.1,126.6$, 129.2, 130.8, 132.4, 132.8; one aromatic carbon peak is hidden. IR (KBr) 3425, 2959, 2923, 2693, 1467, 1383, 1162, 933, 901, 854, 743, $736 \mathrm{~cm}^{-1}$. MS (FAB) m/z (\%) 235 ([M-HCl] $\left.]^{+}, 28\right), 191$ (100).

Free amine $\mathbf{1}$ was obtained by making an aqueous solution of $\mathbf{1} \cdot \mathrm{HCl}$ strongly basic with aqueous $\mathrm{NaOH}$. The basic solution $\left(\mathrm{pH}>11\right.$ ) was extracted with diethyl ether and dried over $\mathrm{MgSO}_{4}$. Evaporation of the solvent gave a yellow solid of free amine $\mathbf{1}$.

1: ${ }^{1} \mathrm{H}$ NMR (DMSO-d $\left.d_{6}\right) \delta 2.23$ (s, $6 \mathrm{H}$ ), 4.32 (s, $2 \mathrm{H}$ ), 7.49 (ddd, $J=8.1,6.3,1.2 \mathrm{~Hz}, 2 \mathrm{H}$ ), 7.54 (ddd, $J=8.4,6.6,1.8 \mathrm{~Hz}, 2 \mathrm{H}$ ), 8.07 (dd, $J=7.8,1.8 \mathrm{~Hz}, 2 \mathrm{H}$ ), 8.47 (d, $J=9.0 \mathrm{~Hz}, 2 \mathrm{H}$ ), 8.55 (s, 1H). ${ }^{13} \mathrm{C}$ NMR (DMSO- $\left.d_{6}\right) \delta 45.0,54.3,124.9,125.0,125.6,127.0,128.7,130.4,130.6,130.9$. IR (KBr) 2960, 2852, 2811, 2762, 1465, 1446, 1342, 1010, 896, 859, $732 \mathrm{~cm}^{-1}$.

A suitable crystal for the solid-state photoreaction was obtained by recrystallization from $i$-PrOH. mp 67-68 ${ }^{\circ} \mathrm{C}$ (lit. ${ }^{3} \mathrm{mp} 65-66^{\circ} \mathrm{C}$ ). Anal. Calcd for $\mathrm{C}_{17} \mathrm{H}_{17} \mathrm{~N}$ : C, 86.77; H, 7.28; N, 5.95. Found: C, 86.48; H, 7.29; N, 5.89.

\section{Preparation of Crystalline Salts}

As a representative procedure, preparation of the salt $\mathbf{1 m u}$ is described below.

\section{Bis[(9-anthrylmethyl)dimethylammonium] muconate (1mu)}

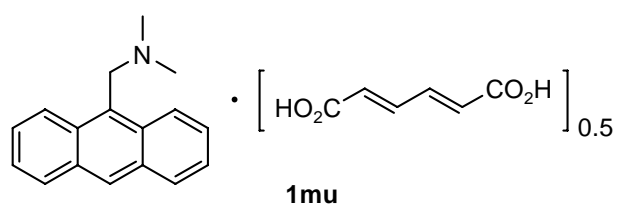

A solution of trans,trans-muconic acid (22.8 $\mathrm{mg}, 0.16 \mathrm{mmol})$ in $\mathrm{MeOH}(20 \mathrm{~mL})$ was added to a solution of 1 (76.0 mg, $0.32 \mathrm{mmol}$ ) in $\mathrm{MeOH}(2 \mathrm{~mL})$ and the mixture was allowed to stand at room temperature in the dark. After two days, yellow crystals precipitated. They were collected by filtration and dried in vacuo to afford yellow prisms of $\mathbf{1 m u}$ (57.0 mg, $0.19 \mathrm{mmol}, 59 \%$ ), $\mathrm{mp}>182{ }^{\circ} \mathrm{C}$ (dec.). The composition $\mathbf{1}$ /acid in the salt was determined on the basis of the ${ }^{1} \mathrm{H}$ NMR and elemental

3 Ihmels, H.; Leusser, D.; Pfeiffer, M.; Stalke, D. Tetrahedron 2000, 56, 6867-6875. 
analysis. $\quad{ }^{1} \mathrm{H}$ NMR (DMSO-d $\left.d_{6}\right) \delta 2.24$ (s, $6 \mathrm{H}$ ), 4.33 (s, $2 \mathrm{H}$ ), 6.29 (dd, $J=11.6,3.2 \mathrm{~Hz}, 1 \mathrm{H}$ ), 7.28 (dd, $J=11.6,3.2 \mathrm{~Hz}, 1 \mathrm{H}$ ), 7.49 (ddd, $J=8.0,6.4,1.2 \mathrm{~Hz}, 2 \mathrm{H}$ ), 7.53 (ddd, $J=8.8,6.8,1.6 \mathrm{~Hz}, 2 \mathrm{H}$ ), 8.06 (dd, $J=8.4,2.0 \mathrm{~Hz}, 2 \mathrm{H}$ ), 8.47 (dd, $J=8.8,1.2 \mathrm{~Hz}, 2 \mathrm{H}$ ), 8.55 (s, $1 \mathrm{H}$ ). IR (KBr) 3447 (br), 3034, 3010, 2261 (br), 1607, 1560, 1344, 1327, 1236, 1193, 1116, 1016, 937, $734 \mathrm{~cm}^{-1}$. Anal. Calcd for $\mathrm{C}_{20} \mathrm{H}_{20} \mathrm{NO}_{2}$ : C, 78.40; H, 6.58; N, 4.57. Found: C, 78.33; H, 6.55; N, 4.57.

All the other salts were prepared by similar procedures. The data used for their characterization are as follows.

(9-Anthrylmethyl)dimethylammonium hydrogen oxalate (10x)

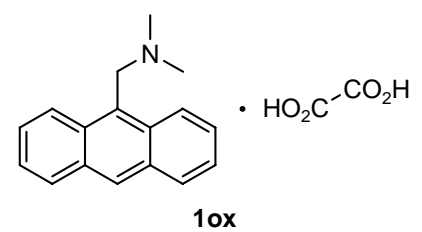

Yellow prisms: $\mathrm{mp}>220{ }^{\circ} \mathrm{C}$ (dec.). ${ }^{1} \mathrm{H}$ NMR $\left(\right.$ DMSO-d $\left._{6}\right) \delta 2.69$ (br, s, $6 \mathrm{H}$ ), 5.11 (br, s, $2 \mathrm{H}$ ), 7.58 (t, $J=7.6 \mathrm{~Hz}, 2 \mathrm{H}), 7.66$ (t, $J=7.6 \mathrm{~Hz}, 2 \mathrm{H}$ ), 8.16 (d, $J=8.4 \mathrm{~Hz}, 2 \mathrm{H}), 8.52$ (d, $J=8.8 \mathrm{~Hz}, 2 \mathrm{H}$ ), 8.75 (s, 1 H). IR (KBr) 3493, 2958, 2922, 2695, 1719, 1701, 1624, 1466, 1405, 1281, 1220, 1195, 1161, 1126, 932, 902, 736, $721 \mathrm{~cm}^{-1}$. Anal. Calcd for $\mathrm{C}_{19} \mathrm{H}_{19} \mathrm{NO}_{4}: \mathrm{C}, 70.14 ; \mathrm{H}, 5.89 ; \mathrm{N}, 4.31$. Found: C, 69.79; H, 5.95; N, 4.32 .

\section{Bis[(9-anthrylmethyl)dimethylammonium] succinate (1su)}

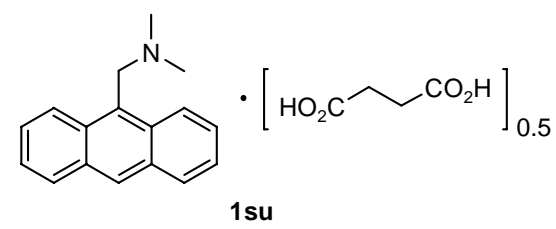

Yellow plates: mp 147-148 ${ }^{\circ} \mathrm{C} . \quad{ }^{1} \mathrm{H}$ NMR (DMSO- $\left.d_{6}\right) \delta 2.26$ (s, $\left.6 \mathrm{H}\right), 2.39$ (s, $2 \mathrm{H}$ ), 4.36 (s, $2 \mathrm{H}$ ), 7.49 (ddd, $J=8.4,6.4,1.2 \mathrm{~Hz}, 2 \mathrm{H}$ ), 7.54 (ddd, $J=8.8,6.4,1.3 \mathrm{~Hz}, 2 \mathrm{H}$ ), 8.07 (d, $J=8.0 \mathrm{~Hz}, 2 \mathrm{H}$ ), 8.47 (d, $J=8.8 \mathrm{~Hz}, 2 \mathrm{H}$ ), 8.56 (s, $1 \mathrm{H}$ ). $\quad$ IR (KBr) 3412 (br), 2957, 2921, 2693, 1560, 1395, 1383, 1162, 932, 902, 743, $736 \mathrm{~cm}^{-1}$. Anal. Calcd for $\mathrm{C}_{38} \mathrm{H}_{40} \mathrm{~N}_{2} \mathrm{O}_{4}: \mathrm{C}, 77.52 ; \mathrm{H}, 6.85 ; \mathrm{N}, 4.76$. Found: C, 77.65; H, 7.08; N, 4.74.

\section{(9-Anthrylmethyl)dimethylammonium hydrogen adipate (1ad)}

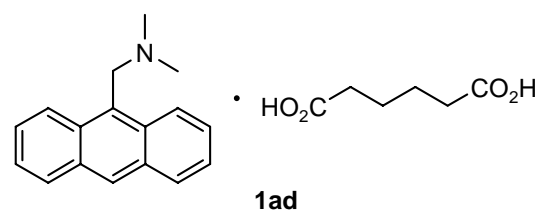

Colorless prisms: mp 132-133 ${ }^{\circ} \mathrm{C} . \quad{ }^{1} \mathrm{H}$ NMR (DMSO- $d_{6}$ ) $\delta 1.48$ (quin, $J=3.9 \mathrm{~Hz}, 4 \mathrm{H}$ ), 2.19 (t, $J$ = 6.9 Hz, $4 \mathrm{H}$ ), 2.24 (s, $6 \mathrm{H}$ ), 4.33 (s, $2 \mathrm{H}$ ), 7.49 (ddd, $J=8.1,7.5,1.2 \mathrm{~Hz}, 2 \mathrm{H}$ ), 7.54 (ddd, $J$ = 8.7, 6.3, $1.5 \mathrm{~Hz}, 2 \mathrm{H}$ ), 8.07 (dd, $J=7.8,2.1 \mathrm{~Hz}, 2 \mathrm{H}), 8.48$ (d, $J=8.7 \mathrm{~Hz}, 2 \mathrm{H}), 8.56$ (s, $1 \mathrm{H})$. IR (KBr) 3411 (br), 3051, 2941, 2447 (br), 1719, 1577, 1405, 1370, 1266, 1181, 1158, 886, 738, $717 \mathrm{~cm}^{-1}$. Anal. Calcd for $\mathrm{C}_{23} \mathrm{H}_{27} \mathrm{NO}_{4}$ : C, 72.42; H, 7.13; N, 3.67. Found: C, 72.32; H, 7.13; N, 3.70. 
Bis[(9-anthrylmethyl)dimethylammonium] fumarate (1fu)

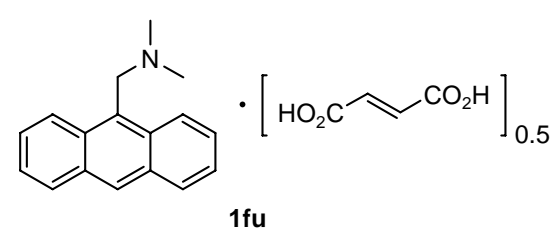

Yellow needles: mp $>149{ }^{\circ} \mathrm{C}$ (dec.). $\quad{ }^{1} \mathrm{H}$ NMR (DMSO-d $\left.d_{6}\right) \delta 2.27$ (s, $6 \mathrm{H}$ ), 4.38 (s, $\left.2 \mathrm{H}\right), 6.58$ (s, 1 H), 7.49 (ddd, $J=8.4,6.8,1.6 \mathrm{~Hz}, 2 \mathrm{H}$ ), 7.54 (ddd, $J=8.4,6.8,1.6 \mathrm{~Hz}, 2 \mathrm{H}$ ), 8.07 (dd, $J=8.0,1.2 \mathrm{~Hz}$, 2 H), 8.47 (dd, $J=8.0,1.2$ Hz, 2 H), 8.56 (s, 1 H). IR (KBr) 3448 (br), 3016, 2946, 1596, 1453, 1339, 1287, 987, 955, 901, 787, 735, $663 \mathrm{~cm}^{-1}$. Anal. Calcd for $\mathrm{C}_{19} \mathrm{H}_{19} \mathrm{NO}_{2}$ : C, 77.79; H, 6.53; N, 4.77. Found: C, 78.03; H, 6.57; N, 4.75.

Bis[(9-anthrylmethyl)dimethylammonium] acetylenedicarboxylate methanol complex (1ac· $\mathrm{MeOH})$

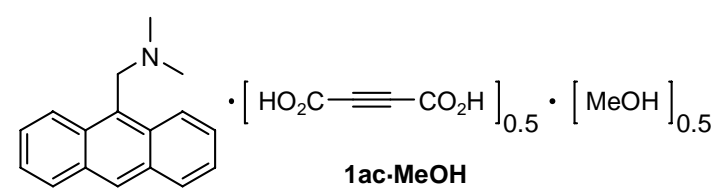

Yellow prisms: mp $112-14{ }^{\circ} \mathrm{C} . \quad{ }^{1} \mathrm{H}$ NMR (DMSO- $\left.d_{6}\right) \delta 2.42$ (s, $\left.6 \mathrm{H}\right), 3.16\left(\mathrm{~s}, \mathrm{CH}_{3}\right.$ of MeOH), 4.63 (br, s, 2 H), 7.52 (t, $J=7.2$ Hz, 2 H), 7.58 (t, $J=7.2$ Hz, 2 H), 8.10 (d, $J=8.4$ Hz, 2 H), 8.49 (d, $J$ $=8.8 \mathrm{~Hz}, 2 \mathrm{H}), 8.63$ (s, $1 \mathrm{H})$. IR (KBr) 3510, 2958, 2694, 1617, 1466, 1364, 1326, 1162, 1125, 989, 978, 933, 902, 781, 743, 736, $673 \mathrm{~cm}^{-1}$. Anal. Calcd for $\mathrm{C}_{19.4} \mathrm{H}_{19.6} \mathrm{NO}_{2.4}:$ C, 76.35; H, 6.47; N, 4.59. Found: C, 76.08; H, 6.53; N, 4.52. Included methanol was not easily lost even by drying in vacuo.

\section{Bis[(9-anthrylmethyl)dimethylammonium] acetylenedicarboxylate (1ac)}

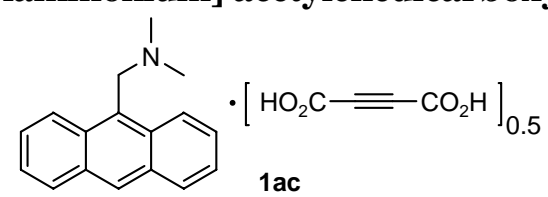

Yellow prisms: mp 137-138 ${ }^{\circ} \mathrm{C} . \quad{ }^{1} \mathrm{H}$ NMR (DMSO-d $\left.\mathrm{d}_{6}\right) \delta 2.39$ (br, s, $6 \mathrm{H}$ ), 4.58 (br, s, $2 \mathrm{H}$ ), 7.52 (t, $J=7.2 \mathrm{~Hz}, 2 \mathrm{H}$ ), 7.58 (t, $J=7.2 \mathrm{~Hz}, 2 \mathrm{H}$ ), 8.10 (d, $J=8.0 \mathrm{~Hz}, 2 \mathrm{H}$ ), 8.49 (d, $J=8.8 \mathrm{~Hz}, 2 \mathrm{H}$ ), 8.62 (s, $1 \mathrm{H}) . \quad$ IR (KBr) 3448, 3031, 2706, 1599, 1449, 1364, 1312, 1276, 1191, 1163, 1124, 978, 933, 896, 784, 729, $667 \mathrm{~cm}^{-1}$. Anal. Calcd for $\mathrm{C}_{19} \mathrm{H}_{18.4} \mathrm{NO}_{2.2}$ : C, 77.11; H, 6.27; N, 4.73. Found: C, 77.05; $\mathrm{H}, 6.50$; N, 4.70; hygroscopic: $0.2 \mathrm{H}_{2} \mathrm{O}$ are retained. 


\section{Spectral Data of the Photochemical Products}

$N, N, N^{\prime}, N^{\prime}$-tetramethyl-5,12[1',2']:6,11[1",2']-dibenzenodibenzo[a,e]cyclooctene-5,11(6H,12 H)- dimethanamine (ht-2)

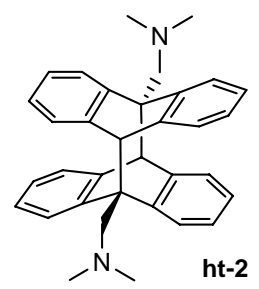

The head-to-tail dimer ht-2 was identified by direct comparison with the authentic sample. ${ }^{2}$ The structure was confirmed by NOESY on the basis of the observed correlation between the bridgehead proton and the methylene proton.

Colorless prisms. $\quad{ }^{1} \mathrm{H}$ NMR $\left(\mathrm{CDCl}_{3}\right) \delta 2.21$ (s, $\left.12 \mathrm{H}\right), 3.68$ (s, $\left.4 \mathrm{H}\right), 3.82(\mathrm{~s}, 2 \mathrm{H})$, 6.77-6.82 (m, $12 \mathrm{H})$, 7.31-7.33 (m, $4 \mathrm{H}) .{ }^{13} \mathrm{C} \mathrm{NMR}\left(\mathrm{CDCl}_{3}\right) \delta$ 46.2, 56.5, 61.8, 63.0, 124.7, 125.2, 127.2, 127.3, 142.8, 143.6. $\mathrm{MS}(\mathrm{FAB}) \mathrm{m} / \mathrm{z}(\%) 471\left([\mathrm{M}+\mathrm{H}]^{+}, 17\right), 235$ (monomer $\left.^{+}, 28\right), 191$ (100).

$N, N, N^{\prime}, N^{\prime}-$ Tetramethyl-6,13-dihydro-7,12[1',2']-benzeno-6,13-ethenobenzo[5,6]cycloocta[1,2 -b] naphthalene-5,12(7H)-dimethanamine (usy-ht-2)

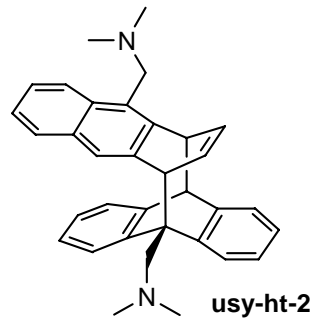

After the irradiation of crystalline salt $\mathbf{1} \mathbf{m u}$ or $\mathbf{1 a c} \cdot \mathbf{M e O H}$, the reaction mixture was neutralized with an aqueous solution of $\mathrm{K}_{2} \mathrm{CO}_{3}$ and extracted with $\mathrm{CH}_{2} \mathrm{Cl}_{2}$. Preparative thin-layer chromatography (silica gel, $\mathrm{CH}_{2} \mathrm{Cl}_{2} / \mathrm{MeOH}$ 9:1) gave a mixture of usy-ht-2 and 1. Crystallization from $\mathrm{MeOH}$ gave pure usy-ht-2.

This compound readily decomposed in chloroform. Colorless prisms. ${ }^{1} \mathrm{H}$ NMR $\left(\mathrm{CD}_{2} \mathrm{Cl}_{2}\right) \delta$ 2.26 (s, 6 H), 2.27 (s, 6 H), 3.54 (d, $J=4.0$ Hz, 1 H), 3.59 (d, $J=12.8 \mathrm{~Hz}, 1 \mathrm{H}$ ), 3.69 (d, $J=13.6 \mathrm{~Hz}, 1$ H), 3.73 (d, $J=13.6 \mathrm{~Hz}, 1 \mathrm{H}$ ), 3.83 (d, $J=12.8 \mathrm{~Hz}, 1 \mathrm{H}$ ), 4.51 (d, $J=11.2 \mathrm{~Hz}, 1 \mathrm{H}$ ), 4.68 (dd, $J=10.8$, $7.2 \mathrm{~Hz}$ ), 5.89 (t, $J=7.6 \mathrm{~Hz}, 1 \mathrm{H}), 6.02$ (td, $J=7.6,0.8 \mathrm{~Hz}, 1 \mathrm{H}), 6.58$ (td, $J=7.6,2.0 \mathrm{~Hz}, 2 \mathrm{H}), 6.82$ (dd, $J=6.0,2.8 \mathrm{~Hz}, 1 \mathrm{H}$ ), 7.13 (s, $1 \mathrm{H}$ ), 7.20 (td, $J=7.6,2.0 \mathrm{~Hz}, 2 \mathrm{H}$ ), 7.25-7.34 (m, $4 \mathrm{H}$ ), 7.54 (dd, $J=8.0,1.8 \mathrm{~Hz}, 1 \mathrm{H}), 7.87$ (dd, $J=6.4,2.4 \mathrm{~Hz}, 1 \mathrm{H}), 8.01(\mathrm{~d}, J=8.4 \mathrm{~Hz}, 1 \mathrm{H}) .{ }^{13} \mathrm{C} \mathrm{NMR}\left(\mathrm{CD}_{2} \mathrm{Cl}_{2}\right) \delta$ 44.6, 45.5, 46.5, 53.7, 56.0, 56.5, 58.3, 62.5, 124.5, 124.9, 125.0, 125.1, 125.2, 125.4, 126.2, 126.5, 126.8, 127.1, 127.3, 127.8, 129.1, 131.5, 132.1, 136.1, 136.7, 142.2, 142.5, 143.5, 143.8, 144.4, 144.9; one aromatic or olefinic carbon peak is hidden. MS (FAB) m/z (\%) $471\left([\mathrm{M}+\mathrm{H}]^{+}, 31\right), 236$ ([monomer $\left.+\mathrm{H}]^{+}, 17\right), 235$ (monomer $\left.^{+}, 16\right), 191$ (100). 


\section{Lepidopterene (3)}

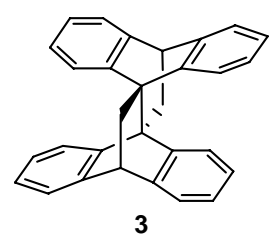

This product was identified by direct comparison with an authentic sample., ${ }^{4,5}$

Colorless prisms. $\quad{ }^{1} \mathrm{H}$ NMR $\left(\mathrm{CDCl}_{3}\right) \delta 2.88(\mathrm{~d}, J=2.9 \mathrm{~Hz}, 4 \mathrm{H}), 4.62(\mathrm{t}, J=2.8 \mathrm{~Hz}, 2 \mathrm{H}), 6.71$ (d, $J=7.5 \mathrm{~Hz}, 4 \mathrm{H}), 6.79$ (td, $J=7.6,1.3 \mathrm{~Hz}, 4 \mathrm{H}), 6.98$ (td, $J=7.3,1.1 \mathrm{~Hz}, 4 \mathrm{H}), 7.32$ (dd, $J=7.2,1.2 \mathrm{~Hz}$, $4 \mathrm{H}) . \quad{ }^{13} \mathrm{C} \mathrm{NMR}\left(\mathrm{CDCl}_{3}\right) \delta 28.8,45.3,53.9,122.6,123.2,125.1,125.4,143.2,143.4 . \quad$ MS (FAB) $\mathrm{m} / \mathrm{z}(\%) 382\left(\mathrm{M}^{+}, 47\right), 191$ (100). $\quad$ HRMS (FAB) calcd. 382.1721 for $\mathrm{C}_{30} \mathrm{H}_{22}$, found 382.1718.

\section{1,2,7,8-Tetrahydro-2a,7[1',2']:8,12b[1",2']-dibenzenodibenzo[a,e]cyclobuta[c]cyclooctene} (4)

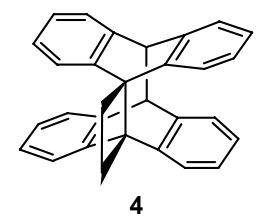

This product was identified by direct comparison with an authentic sample. ${ }^{6}$ Irradiation of a solution of 1,2-bis(anthryl)ethane in chlorobenzene under Ar atmosphere gave $\mathbf{4}$ quantitatively. After the solvent was evaporated, the residue was recrystallized from $\mathrm{CHCl}_{3}$ to give pure 4. ${ }^{1} \mathrm{H} \mathrm{NMR}$ $\left(\mathrm{CDCl}_{3}\right) \delta 2.99$ (s, $\left.4 \mathrm{H}\right), 4.50$ (s, $\left.2 \mathrm{H}\right), 6.82$ (td, $\left.J=6.9,1.2 \mathrm{~Hz}, 4 \mathrm{H}\right), 6.89$ (td, $J=7.2,1.5 \mathrm{~Hz}, 4 \mathrm{H}$ ), 6.95 (dd, $J=6.9,1.5 \mathrm{~Hz}, 4 \mathrm{H}$ ), 7.09 (dd, $J=4.5,1.2 \mathrm{~Hz}, 4 \mathrm{H}) . \quad{ }^{13} \mathrm{C} \mathrm{NMR}\left(\mathrm{CDCl}_{3}\right) \delta$ 15.8, 53.7, 61.6, 123.1, 125.3, 125.4, 127.5, 143.0, 143.5 .

\section{9,10-Dihydro-9-methylanthracene (5)}

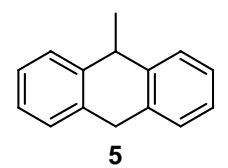

This product was identified by direct comparison with an authentic sample. ${ }^{7}$

A colorless solid. ${ }^{1} \mathrm{H}$ NMR $\left(\mathrm{CDCl}_{3}\right) \delta 1.42(\mathrm{~d}, J=6.9 \mathrm{~Hz}, 3 \mathrm{H}), 3.88(\mathrm{~d}, J=18.3 \mathrm{~Hz}, 1 \mathrm{H}), 4.04$ (q, $J=7.2 \mathrm{~Hz}, 1 \mathrm{H}), 4.12$ (d, $J=18.3 \mathrm{~Hz}, 1 \mathrm{H}), 7.14-7.31(\mathrm{~m}, 8 \mathrm{H}) .{ }^{13} \mathrm{C} \mathrm{NMR}\left(\mathrm{CDCl}_{3}\right) \delta 23.5,35.1$, 41.1, 125.9, 126.3, 126.8, 127.6, 135.7, 141.7. MS (EI) m/z (\%) 194 (M+10), 179 (100), 178 (38), 89 (12).

\footnotetext{
${ }^{4}$ Fernandez, M.-J.; Gude, L.; Lorente, A. Tetrahedron Lett. 2001, 42, 891-893.

${ }^{5}$ Deardurff, L. A.; Alnajjar, M. S.; Camaioni, D. M. J. Org. Chem. 1986, 51, 3686-3693.

6 Becker, H.-D.; Elebring, T.; Sandros, K. J. Org. Chem. 1982, 47, 1064-1068.

7 Takagi, M.; Hirabe, T.; Nojima, M.; Kusabayashi, S. J. Chem. Soc., Perkin Trans. 1 1983, 1311-1314.
} 


\section{Dissociation Kinetics of usy-ht-2}

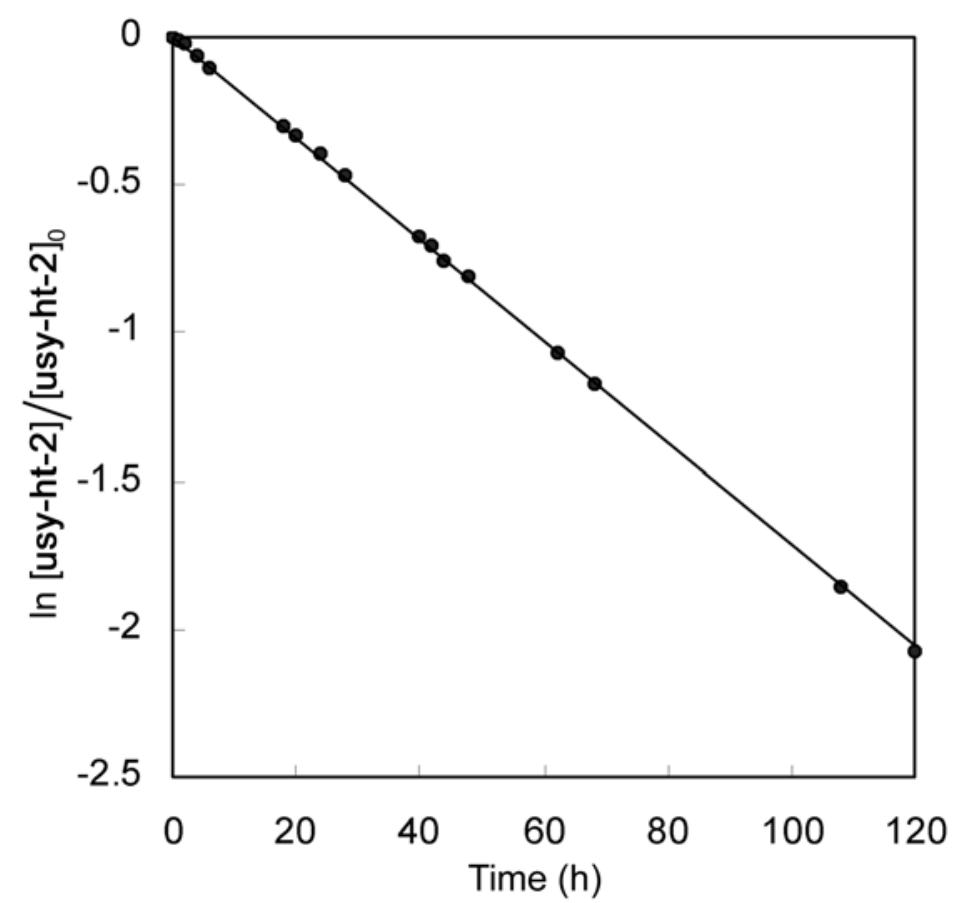

Figure S1. Semilogarithmic plot of [usy-ht-2]/[usy-ht-2 $]_{0}$ against time at $40{ }^{\circ} \mathrm{C}$.

\section{X-ray Crystal Structure Analysis}

The diffraction data were collected on a Rigaku RAXIS-RAPID Imaging Plate diffractometer with graphite monochromated Mo-Ka radiation to a maximum $2 \theta$ value of $55.0 \AA$. The data were corrected for Lorentz and polarization effects. The structure was solved by direct methods and expanded using Fourier techniques. The non-hydrogen atoms were refined anisotropically. The hydrogen atoms were located on the geometrically calculated position with ideal distances $\left(\mathrm{CH}_{3} 0.96 \AA, \mathrm{CH}_{2} 0.97 \AA, \mathrm{CH}\right.$ $0.98 \AA$, aromatic and olefinic $\mathrm{CH} 0.93 \AA$, NH $0.89 \AA, \mathrm{OH} 0.82 \AA$ ), and their parameters were not refined. All calculations were performed using the teXsan crystallographic software package of Molecular Structure Corporation. The crystal structure parameters for 1ox, 1su, 1ad, 1mu, 1ac· MeOH, 1ac and usy-ht-2 are summarized in Table S1. 
Table S1. Crystallographic parameters

\begin{tabular}{ccccc}
\hline & $\mathbf{1 0 x}$ & $\mathbf{1 s u}$ & $\mathbf{1 a d}$ & $\mathbf{1 m u}$ \\
\hline chemical formula & $\mathrm{C}_{17} \mathrm{H}_{18} \mathrm{~N}^{+}$ & $\mathrm{C}_{17} \mathrm{H}_{18} \mathrm{~N}^{+}$ & $\mathrm{C}_{17} \mathrm{H}_{18} \mathrm{~N}^{+}$ & $\mathrm{C}_{17} \mathrm{H}_{18} \mathrm{~N}^{+}$ \\
& $\cdot \mathrm{C}_{2} \mathrm{HO}_{4}{ }^{-}$ & $\cdot 0.5\left(\mathrm{C}_{4} \mathrm{H}_{4} \mathrm{O}_{4}\right)^{-}$ & $\cdot \mathrm{C}_{6} \mathrm{H}_{9} \mathrm{O}_{4}{ }^{-}$ & $\cdot 0.5\left(\mathrm{C}_{6} \mathrm{H}_{4} \mathrm{O}_{4}\right)^{-}$ \\
formula weight & 325.36 & 294.37 & 381.47 & 306.38 \\
crystal system & monoclinic & monoclinic & monoclinic & monoclinic \\
space group & $P 2_{1} / c$ & $P 2_{1} / c$ & $C 2 / c$ & $P 2_{1} / c$ \\
$a(\AA)$ & $8.545(2)$ & $10.1556(4)$ & $23.402(1)$ & $10.124(4)$ \\
$b(\AA)$ & $17.764(5)$ & $8.8798(4)$ & $14.6147(6)$ & $14.703(5)$ \\
$c(\AA)$ & $11.252(2)$ & $16.9199(9)$ & $17.5801(9)$ & $11.485(4)$ \\
$\beta($ deg$)$ & $110.089(3)$ & $89.478(1)$ & $137.4727(9)$ & $110.43(2)$ \\
$V\left(\AA^{3}\right)$ & $1604.0(7)$ & $1525.8(1)$ & $4064.2(3)$ & $1602(3)$ \\
$Z$ & 4 & 4 & 8 & 4 \\
$D_{\text {calc }}\left(\mathrm{g} / \mathrm{cm}{ }^{3}\right)$ & 1.347 & 1.281 & 1.247 & 1.270 \\
$R$ & 0.0452 & 0.0567 & 0.0462 & 0.0446 \\
$R_{\mathrm{w}}$ & 0.1084 & 0.1390 & 0.1365 & 0.1358 \\
$\mathrm{GOF}$ & 1.128 & 1.322 & 1.217 & 1.334 \\
nof & 3066 & 3005 & 3408 & 3027 \\
no. of parameters used & 217 & 199 & 253 & 208 \\
\hline
\end{tabular}

\begin{tabular}{cccc}
\hline & 1ac·MeOH & 1ac & usy-ht-2 \\
\hline chemical formula & $2\left(\mathrm{C}_{17} \mathrm{H}_{18} \mathrm{~N}^{+}\right)$ & $\mathrm{C}_{17} \mathrm{H}_{18} \mathrm{~N}^{+}$ & $\mathrm{C}_{34} \mathrm{H}_{34} \mathrm{~N}_{2}$ \\
& $\cdot \mathrm{C}_{4} \mathrm{O}_{4}{ }^{2-} \cdot \mathrm{CH}_{4} \mathrm{O}$ & $\cdot 0.5\left(\mathrm{C}_{4} \mathrm{O}_{4}\right)^{-}$ & \\
formula weight & 616.76 & 292.36 & 470.66 \\
crystal system & monoclinic & monoclinic & monoclinic \\
space group & $P 2_{1} / a$ & $P 2_{1} / n$ & $P n$ \\
$a(\AA)$ & $10.9945(8)$ & $10.5311(5)$ & $9.7776(5)$ \\
$b(\AA)$ & $15.532(1)$ & $11.2818(7)$ & $13.6558(5)$ \\
$c(\AA)$ & $19.610(1)$ & $13.3134(7)$ & $9.8864(5)$ \\
$\beta(\mathrm{deg})$ & $103.6889(9)$ & $108.860(2)$ & $101.0143(6)$ \\
$V\left(\AA \AA^{3}\right)$ & $3253.5(4)$ & $1496.8(1)$ & $1295.7(1)$ \\
$Z$ & 4 & 4 & 2 \\
$D_{\text {calc }}\left(\mathrm{g} / \mathrm{cm}{ }^{3}\right)$ & 1.259 & 1.297 & 1.206 \\
$R$ & 0.0800 & 0.0634 & 0.0505 \\
$R_{\mathrm{w}}$ & 0.2146 & 0.1805 & 0.1505 \\
$\mathrm{GOF}$ & 1.769 & 1.183 & 1.373 \\
no. of reflections used & 5440 & 2070 & 2940 \\
no. of parameters used & 415 & 199 & 325 \\
\hline
\end{tabular}




\section{7. ${ }^{1} \mathrm{H}$ and ${ }^{13} \mathrm{C}$ NMR Charts of usy-ht-2}
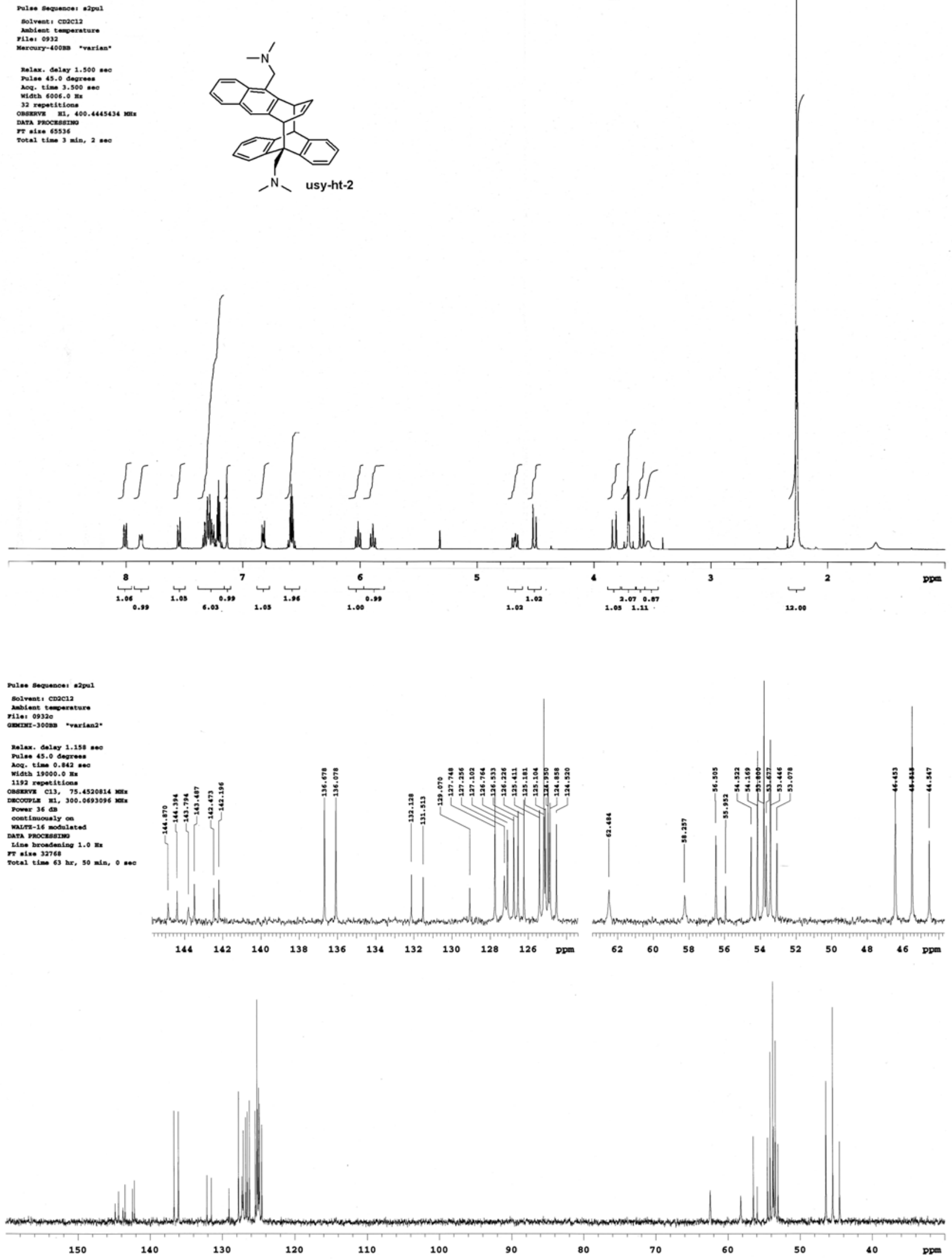

${ }^{1} \mathrm{H}$ and ${ }^{13} \mathrm{C}$ NMR spectra of usy-ht-2 